\title{
Strength of silicon nanolayers under tensile loading
}

\author{
Iskandarov A.M. ${ }^{1 \dagger}$, Umeno Y. ${ }^{1}$ \\ †albert@ulab.iis.u-tokyo.ac.jp
}

${ }^{1}$ Institute of Industrial Science, The University of Tokyo, 4-6-1 Komaba Meguro-ku, 153-8505 Tokyo

Tensile strength of silicon slabs is estimated by means of classical molecular dynamics simulation. Effects of temperature and surface defects, namely vacancies and linear steps, on the strength are examined. It is found that the presence of vacancies on the slab's surface decreases the critical strain, whereas the change of their concentration does not cause its significant changes. In contrast, increasing the temperature and surface step height gradually decreases the critical strain.

Keywords: silicon, strength, tension, defects.

\section{Introduction}

Mechanical response of nanostructures can be much different from that in bulk. Main reason arises from nanoscale dimensions of these objects that restrict operation of conventional deformation mechanisms, such as dislocation multiplication through Frank-Read sources [1]. Thus, nanostructures can sustain huge strains that make them attractive, for instance, for elastic strain engineering, tuning their properties by applying elastic strain [2]. Due to lack of defects in these nanostructures, their strength is limited by defect nucleation at a free surface and their further propagation $[3,4]$.

Defect nucleation at free surfaces of Si nanostructures has been widely studied experimentally $[5,6]$ and theoretically [7-10]. Relative complexity of surface defect nucleation in $\mathrm{Si}$ is dictated by its diamond lattice and presence of two sets of $\{111\}$ slip planes: the shuffle and glide sets [11-13]. Godet et al. confirmed using molecular dynamics (MD) simulations that each of these two dislocation sets can be activated in Si slabs from surfaces under tension at particular conditions [14]. At high temperatures and low tensile stress nucleation of dislocations in a glide set was observed, while at low temperatures and high tensile stress shuffle dislocation nucleation was reported [15]. In a different study Godet et al. examined mechanism of mechanical failure of (001) surface oriented Si nanolayer on tensile direction [8]. It was found that, besides surface dislocations, cracks can nucleate and propagate in material when straining it in tension along $<110\rangle$ direction. This effect was confirmed within a temperature range up to $900 \mathrm{~K}$. In work by Kang et al. similar result was obtained in MD simulation of tension of a [110] oriented nanowire. At a higher temperature, viz $1000 \mathrm{~K}$, shear on a $\{111\}$ plane was observed [16]. Moreover, when a nanowire of a smaller radius (less than $4 \mathrm{~nm}$ ) was tested, no crack nucleation was observed and the nanowire always underwent extensive slip in the same $\{111\}$ plane. Therefore we may conclude that mechanical response of $\mathrm{Si}$ nanostructures is very complicated and affected by various external factors, like temperature, surface defects, loading condition, etc.
In the present work we study the effect of sufrace vacancies and surface steps on critical tensile strain of Si nanoslabs. Tensile orientation was chosen to be along $<110>$ crystallographic direction because according to Godet et al. Si can sustain largest strain when subjected to tension in this direction [6]. We examined the effect of surface vacancy concentration and height of surface steps on the critical strain.

\section{Calculation setup}

In the present study we employed classical MD simulations using LAMMPS package [17]. All simulations were based on initial rectangular silicon slab model of size $230 \times 31 \times 130 \AA$, with [ $\overline{1} 10]$ crystallographic direction being aligned along the $x$ axis, [110] — along the $y$ axis, and [001] — along the $z$ axis (see fig.1). Atoms on free [001] surfaces were $p(2 \times 1)$ reconstructed. Along all three directions periodic boundary conditions were used. However, along the $z$ axis vacuum gap was introduced thick enough (around $20 \AA$ ) to avoid interaction of slab's surfaces across periodic $z$ boundaries. Tension was applied by uniformly increasing simulation cell size along the $x$ axis till the moment of mechanical failure. The cell shape was kept rectangular, and the cell size along $z$ direction was kept constant. The latter did not restrict slab's shrinkage along the $z$ direction due to Poisson effect because of the presence of the free surface. To allow material to shrink along the $y$ axis too we allowed cell size along this direction to change to keep on average zero $\sigma_{\mathrm{yy}}$ stress component. Temperature varied in the range from $0 \mathrm{~K}$ to $1200 \mathrm{~K}$. In each calculation the system was initially equilibrated for 20 ps at a given temperature before applying tension. Critical strain was determined by the position of maximum in the strainstress curve. For each calculation condition we performed 10 independent runs with different initial atomic velocity distributions in order to get an averaged value of critical strain. This way of critical strain/stress estimation was successfully applied by us for ideal shear strength evaluation at elevated temperatures [18]. 


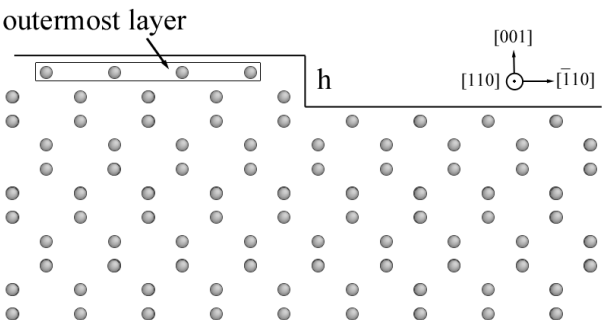

Fig. 1. Atomic structure of Si slab near surface step of height $h$. Outermost atomic layer is pointed from which atoms were removed to introduce surface vacancies.

The effect of defects on critical tensile strain was studied in similar slab structures obtained from initial one after introducing defects. We considered two types of defects, namely surface vacancies and linear steps on surface along [110] direction. Vacancy concentration on surface is measured as a percentage of randomly removed atoms in outermost atomic layer. Sampled concentration values are 0 , 10,20 , and $30 \%$. We considered four step heights, viz $h$ (as shown in fig. 1$), 2 h, 3 h$, and $4 h$.

Interatomic interactions were described by the StillingerWeber potential [19]. This choice was dictated by our previous empirical potential analysis for $\mathrm{Si}$, which can be found elsewhere [20].

\section{Results and discussion}

In the case of traction along [ $1 \overline{1} 10]$ direction, according to Godet et al., at $\mathrm{T} \leq 900 \mathrm{~K} \mathrm{Si}$ slab undergoes mechanical failure at critical strain by means of crack nucleation at the [001] surface and its propagation [8]. In our calculations in the same temperature range we also observed only crack nucleation in configurations with defects and perfect ones. In fig. 2 we illustrate such crack cleavage at $0 \mathrm{~K}$ that happened with perfect slab at $\varepsilon_{c}=25.3 \%$. At $1200 \mathrm{~K}$ (and sometimes at $900 \mathrm{~K}$ as well), however, we observed shear failure, when sliding occurred on $\{111\}$ planes. Qualitatively this behavior is also in good agreement with MD simulations of tension of [110]-oriented Si nanowire performed by Kang et al., who obtained brittle fracture of the nanowire at $300 \mathrm{~K}$ and ductile one at $1000 \mathrm{~K}[16]$.

In the case of slab with introduced step of $h$ height at some temperatures our results can be directly compared with those derived by Godet et al. [7]. We summarize them in tab.1. We can see good agreement between obtained values of critical strain at all temperatures, which confirms validity of our approach of simulation result analysis.

Table 1.

Estimated values of critical tensile strain (\%) of Si slab with linear surface step of height $h$

\begin{tabular}{|c|c|c|}
\hline Temperature, K & Godet et al.[7] & Our work \\
\hline 0 & $24.1 \pm 0.5$ & $23.2 \pm 0.4$ \\
\hline 300 & $14.3 \pm 0.5$ & $14.5 \pm 0.3$ \\
\hline 900 & $12.0 \pm 0.5$ & $12.3 \pm 0.5$ \\
\hline
\end{tabular}

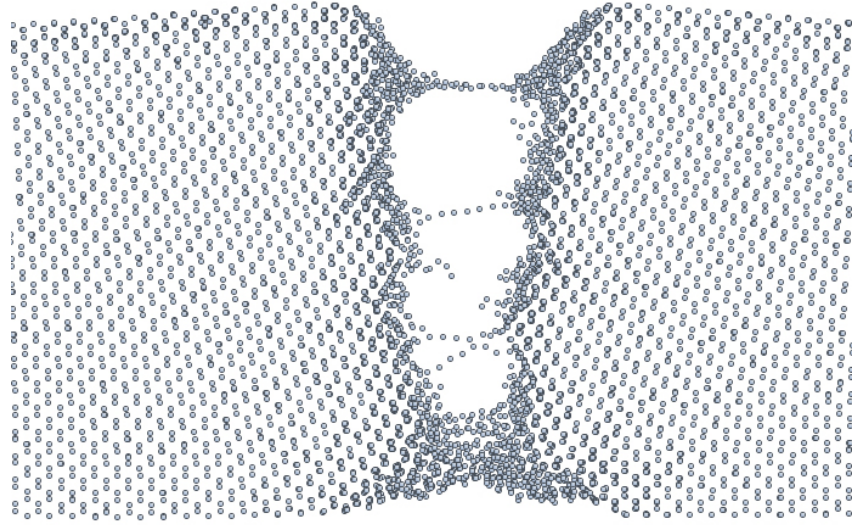

a

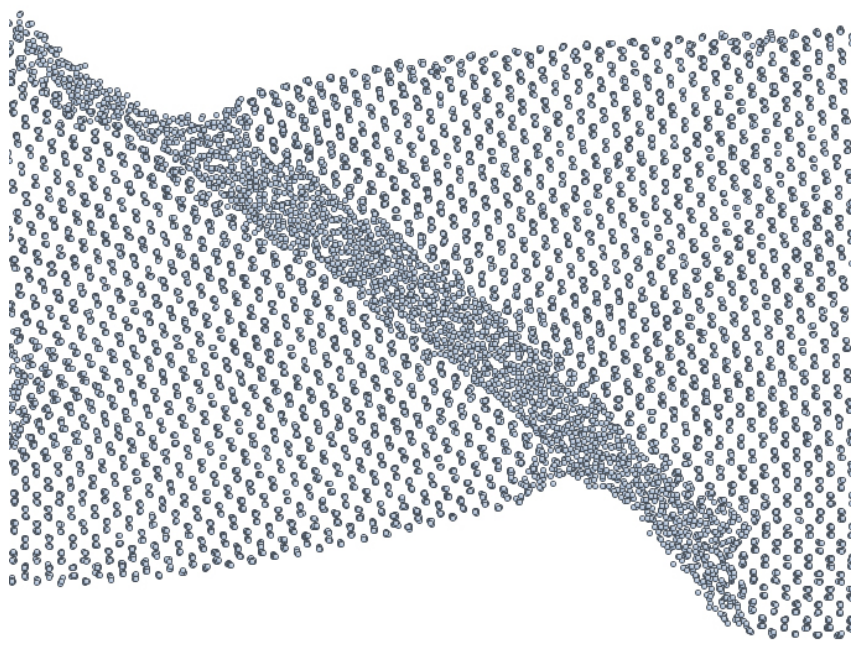

b

Fig. 2. (a) Crack cleavage on (001) surface of Si slab after its nucleation on top surface at critical strain $\varepsilon_{c}=25.2 \%$ and $0 \mathrm{~K}$, and (b) local slip taking place at $\varepsilon_{c}=12.3 \%$ and $1200 \mathrm{~K}$.

In fig. 3 we present dependence of critical tensile strain as a function of temperature for the perfect slab and slabs with three surface vacancy concentrations. All four types of slabs exhibit decrease of critical strain when temperature is increased. Indeed, the smallest values of $\varepsilon \approx 12.3 \%$ in all four cases is obtained at $1200 \mathrm{~K}$. The same critical strain obtained at this temperature indicates that at high temperatures temperature is a dominant factor controlling the onset of mechanical failure compared to surface vacancies. At low temperature region, however, there is notable difference between curves for perfect slab and slabs with vacancies introduced on surfaces. The presence of vacancies on surfaces at low temperatures decreases critical strain from $25.2 \%$ down to $21.3 \%$, particular vacancy concentration does not influence result much, at least for the sampled concentrations. There is however, possibly, some critical vacancy concentration (between 0 and 10\%), below which critical shear strain is around that for perfect slab.

Similarly to the case of slabs with vacancies on surfaces, reduction of $\varepsilon_{c}$ with temperature was obtained for all step heights (see fig.4). However, in contrast, gradual decrease of critical strain at each temperature was found when the height of the steps increases from $0 h$ to $4 h$. Indeed, at $1200 \mathrm{~K}$, while for all surface vacancy concentrations $\varepsilon_{c}=12.5 \%$, value 


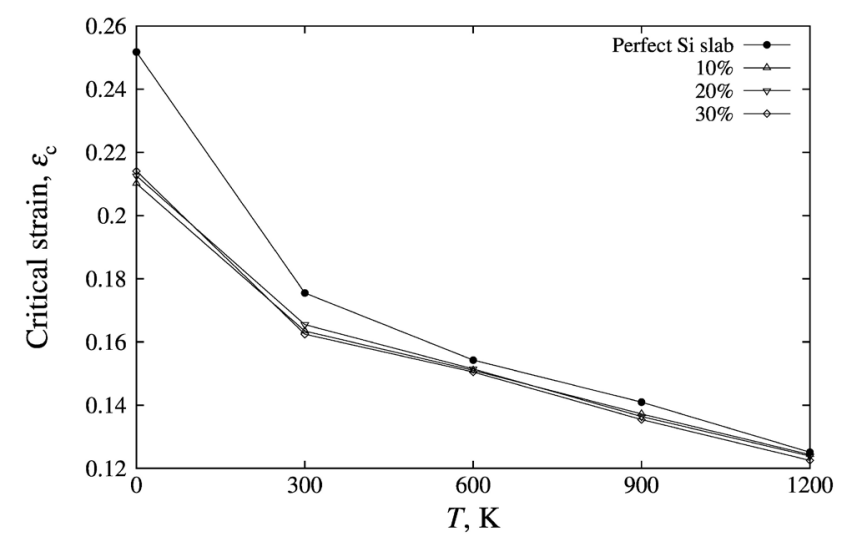

Fig. 3. Critical tensile strain as a function of temperature for perfect Si slab, and slabs with 10,20, and $30 \%$ of removed surface atoms.

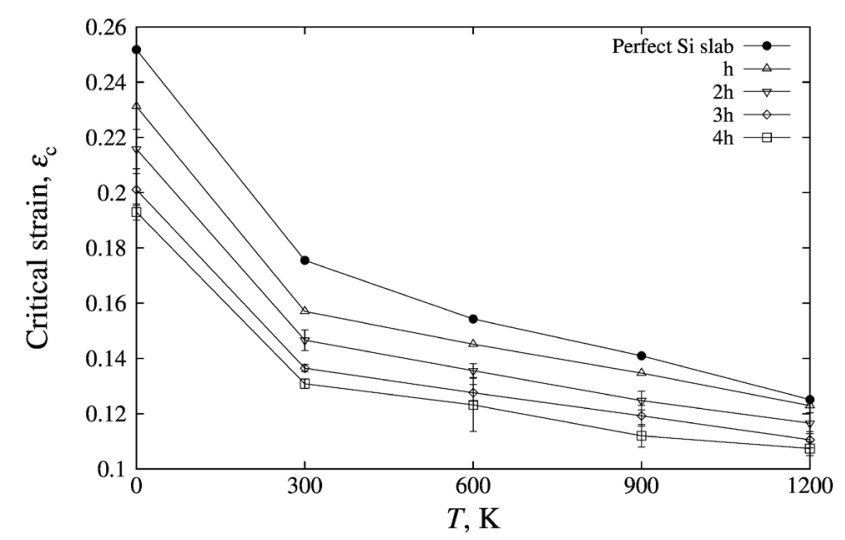

Fig. 4. Critical tensile strain as a function of temperature for perfect Si slab, and slabs with linear steps of $h, 2 h, 3 h$, and $4 h$ height.

of $\varepsilon_{c}$ gradually decreases at this temperature down to $10.7 \%$ when step height increases. In the low temperature region we observe similar behavior of a more pronounced effect of defects on critical strain as we did in the case of surface vacancies. It is interesting that the curve for $2 h$ step height is closest to those for slabs with surface vacancies shown in fig.3. This means that the reduction of strength due to the presence of vacancies on free surface is comparable with that of $2 h$ height surface step.

\section{Summary}

We performed MD simulation of tensile deformation of Si slabs in a wide range of temperatures. The study of the influence of surface defects on critical strain showed that they significantly weaken the slabs, the effect being most pronounced in the low temperature region. Higher temperature tends to decrease the difference between results. Indeed, at $0 \mathrm{~K}$ all critical strains fall in a $6 \%$ range, while at $1200 \mathrm{~K}$ in a range of $2 \%$. In absolute values the most significant decrease of critical strain due to temperature was observed for the perfect slab, from $25.2 \%$ to $12.5 \%$.

As for a qualitative difference of effect to critical strain between the considered defect types, we can recall that within the sampled vacancy concentrations and step heights there is gradual decrease of critical strain at any given temperature with increase of step height, while the change of vacancy concentration does not affect results in contrast to the salient decrease of critical strain due to removing $10 \%$ of surface atoms from a perfect slab.

One of the authors (AI) acknowledges financial support from Russian Foundation for Basic Research, grant \#12-0231519 mol_a.

\section{References}

1. T. Zhu and J. Li. Prog. Mater. Sci. 55, 710 (2010).

2. T. Zhu, J. Li, S. Ogata, S. Yip. MRS Bull. 34, 167 (2009).

3. H. Bei, Y.F. Gao, S. Shim, E.P. George, and G. M. Pharr. Phys. Rev. B. 77, 060103 (R) (2008).

4. A.M. Minor, S.A. S. Asif, Z. Shan, E.A. Stach, E. Cyrankowski, T. J. Wyrobek, and O. L. Warren. Nature Materials. 5, 697 (2006).

5. J. Rabier and J. L. Demenet, Phys. Stat. Sol. (a). 202, 944 (2005).

6. K. Asaoka, T. Umeda, S. Arai, and H. Saka. Mat. Sci. Eng. A. 400-401, 93 (2005).

7. J. Godet, L. Pizzagalli, S. Brochard, and P. Beauchamp. Scripta Mater. 47, 481 (2002).

8. J. Godet, L. Pizzagalli, S. Brochard, and P. Beauchamp. Phys. Rev. B. 70, 054109 (2004).

9. S. Izumi and S. Yip. J. Appl. Phys. 104, 033513 (2008).

10. K. Kang and W. Cai. Phil. Mag. 87, 2169 (2007).

11. J. Hornstra. J. Phys. Chem. Solids. 5, 129 (1958).

12. J. Justo, M. de Koning, W. Cai, and V. Bulatov. Phys. Rev. Lett. 84, 2172 (2000).

13. L. Pizzagalli and P. Beachamp. Phil. Mag. Lett. 84, 729 (2004).

14. J. Godet, P. Hirel, S. Brochard, and L. Pizzagalli. J. Appl. Phys. 105, 026104 (2009).

15. K. Shima, S. Izumi, and S. Sakai. J. Appl. Phys. 108, 063504 (2010).

16. K. Kang and W. Cai. Int. J. Plast. 26, 1387 (2010).

17. http://lammps.sandia.gov/

18. A.M. Iskandarov, S.V. Dmitriev, and Y. Umeno. Phys. Rev. B. 84, 224118 (2011).

19. F.H. Stillinger and T.A. Weber. Phys. Rev. B. 31, 5262 (1985).

20. A. M. Iskandarov and Y. Umeno. Fundamental problems of material science. 9(1), 89 (2012).96.30.nd 88.50.gj 